\title{
Inhibitory effect of RNA-mediated knockdown of zinc finger protein 91 pseudogene on pancreatic cancer cell growth and invasion
}

\author{
WEIYI HUANG, NING LI, JIONG HU and LEI WANG \\ Department of Oncology, Shanghai Jiaotong University Affiliated Shanghai General Hospital, Shanghai 200085, P.R. China
}

Received April 3, 2015; Accepted May 23, 2016

DOI: $10.3892 / \mathrm{ol} .2016 .4794$

\begin{abstract}
Worldwide, human pancreatic cancer is a rare malignancy with a poor prognosis. Long non-coding RNAs (lncRNAs) are known to have a crucial role in cancer occurrence and progression; however, the role of pseudogene-expressed IncRNAs, a major type of lncRNA, have not been thoroughly analyzed in cancer. Therefore, the present study focused on zinc finger protein 91 pseudogene (ZFP91-P). ZFP91-P expression was initially detected in two pancreatic cancer cell lines by reverse transcription-quantitative polymerase chain reaction (RT-qPCR) and the highest expression of ZFP91-P was found in the BXPC-3-H cell line. Subsequently, BXPC-3-H cells were transfected with ZFP91-P short hairpin RNA (shRNA) using a plasmid vector and termed shZFP91-P. Cells transfected with negative control plasmid vector were termed shCon. MTT and Transwell assays were performed to analyze the proliferation and migration of BXPC-3-H cells, respectively, and western blotting was used to detect epithelial-mesenchymal transition markers, including vimentin and $\beta$-catenin. The present study showed that depletion of ZFP91-P markedly decreased pancreatic cancer cell proliferation and inhibited cell migration capacity. In addition, the expression of $\beta$-catenin increased while vimentin expression decreased. The current findings suggest that high expression of ZFP91-P promotes the migration of BXPC-3-H cells and may be a novel marker for early diagnosis for pancreatic cancer.
\end{abstract}

\section{Introduction}

Pancreatic cancer is one of the most lethal neoplastic diseases with an overall 5-year survival rate of $\sim 7 \%$ in USA (1). It is the fourth leading cause of cancer-related mortality in the USA, and its mortality rate of $\sim 7 \%$ has not decreased in Western

Correspondence to: Mr. Weiyi Huang, Department of Oncology, Shanghai Jiaotong University Affiliated Shanghai General Hospital, 100 Haining Road, Shanghai 200085, P.R. China

E-mail: weiyihuangdr@163.com

Key words: zinc finger protein 91 pseudogene, pancreatic cancer, lentivirus, cell invasion, siRNA, target therapy countries over the past few decades $(1,2)$. Advanced stage pancreatic cancer with a $<5$-year survival rate is more common in elderly individuals than in younger patients $(3,4)$. To better solve this health problem, numerous studies are focusing on the molecular mechanism of pancreatic cancer occurrence in order to develop novel treatment strategies (5-8).

Non-coding RNAs (ncRNAs) were previously considered to be transcriptional noise, however, they have more recently been proven to have a pivotal role in cellular development and various pathologies. Long ncRNAs (lncRNAs), which have a length of $>200 \mathrm{nt}$, are a major group of ncRNAs that can be classified into five categories: Sense, antisense, bidirectional, intronic and intergenic (9). IncRNAs are involved in almost every step of the life cycle of genes and regulate diverse functions (10). Pseudogenes have been emerging as a novel class of lncRNAs and have shown to be important regulatory molecules involved in cancer (11). Welch et al identified 309 pseudogenes with significant differential expression in breast cancer (12). Several transcribed pseudogenes, such as phosphatase and tensin homolog pseudogene 1 (PTENP1), KRAS proto-oncogene, GTPase pseudogene 1 (KRASPI)and POU class 5 homeobox 1 (OCT4)-pg4, have been known to promote tumor progression $(13,14)$. In addition, zinc finger family genes, such as zinc finger protein (ZFP)185, have been revealed to have a strong inverse correlation with prostate cancer progression (15). It has been reported that ZFP91 has a role in hematopoietic repopulating cells (16) and cell proliferation or anti-apoptosis (17). Furthermore, ZFP91 mRNA expression levels have been demonstrated to be upregulated in the gastric tissue of patients with non-alcoholic steatohepatitis (18). However, to the best of our knowledge, no study to date has reported the role of ZFP91 pseudogenes (ZFP91-Ps) in pancreatic cancer and the cellular function of ZFP91-P remains elusive.

The RNA interference (RNAi) technique, as a loss-of-function assay, provides a novel approach for investigating the molecular mechanism of cancer occurrence $(19,20)$. In the present study, ZFP91-P expression was specifically knocked down in human pancreatic cancer cells by constructing lentivirus-mediated short hairpin RNA (shRNA). Subsequently, cell migration was evaluated using a scratch and Transwell migration assay, cell proliferation was evaluated using an MTT assay, and the molecular mechanism of ZFP91-P in pancreatic cancer occurrence was determined. 


\section{Materials and methods}

Cell culture. Human pancreatic cancer cell lines (BXPC-3 and BXPC-3-H) were obtained from the Type Culture Collection of the Chinese Academy of Sciences (Shanghai, China). The BXPC-3-H cell line was generated in our laboratory by screening and verifying high metastatic potential BXPC-3 cells by monoclonal and transwell assays. The 293T human embryonic kidney cell line was purchased from the Cell Bank of Chinese Academy of Sciences (Shanghai, China). Cells were cultured in Dulbecco's modified Eagle medium (Hyclone; GE Healthcare Life Sciences, Logan, UT, USA) with $10 \%$ fetal bovine serum (FBS) and were maintained at $37^{\circ} \mathrm{C}$ in a $5 \% \mathrm{CO}_{2}$ humidified atmosphere.

Lentiviral vector construction. The shRNA sequence (5'-GGGCTGCAGATCTAGTCTTCACTCGAGTGAAG ACTAGATCTGCAGCCC-3') was designed to target human ZFP91-P gene (NR_024380; www.ncbi.nlm.nih.gov). The control shRNA sequence was: 5'-CTAGCCCGGCCAAGG AAGTGCAATTGCATACTCGAGTATGCAATTGCACTT CCTTGGTTTTTTGTTAAT-3'. The shRNA sequences were designed by the present authors, and synthesized by Genewiz, Inc. (Suzhou, China). The target and control shRNAs were annealed, and ligated into the Nhe I/Pac I (NEB, Ipswich, MA, USA)-linearized pFH-L vector (Shanghai Hollybio, Shanghai, China), and termed pFH-L-shZFP91-P and pFH-L-shCon, respectively.

Lentiviral packaging and cell infection. The reconstructed plasmids were transfected into $293 \mathrm{~T}$ cells with $\mathrm{pCMV} \Delta \mathrm{R} 8.92$ and pVSVG-I helper plasmids (Shanghai Hollybio) using Lipofectamine 2000 (Invitrogen; Thermo Fisher Scientific, Inc.). After transfection of $72 \mathrm{~h}$ at $37^{\circ} \mathrm{C}$ in a humidified incubator containing $5 \% \mathrm{CO}_{2}$, supernatants containing Lv-shZFP91-P or Lv-shCon were harvested by purification and precipitation. Then, BXPC-3-H cells $(50,000$ cells/well) were seeded in 6-well plates and transduced with Lv-shZFP91-P or Lv-shCon at a multiplicity of infection of 20. After 4 days infection, cells were observed under a fluorescence microscope (Olympus Corporation, Tokyo, Japan). As the pFH-L lentiviral vector carries a green fluorescence protein (GFP) reporter, the infection efficiency was determined by counting the number of GFP-positive cells compared to total cells.

Reverse transcription-quantitative polymerase chain reaction (RT- $q P C R)$. Total RNA was extracted from cells using TRIzol reagent (Invitrogen; Thermo Fisher Scientific, Inc.) and synthesized into cDNA with random primers, according to the manufacturer's protocol (Fermantas; Thermo Fisher Scientific, Inc.). The reaction system of reverse transcription was as follows: $2 \mu \mathrm{g}$ Total RNA, $1 \mu \mathrm{l}$ oligo dT $(0.5 \mu \mathrm{g} / \mu \mathrm{l})$, $4 \mu 1$ M-MLV buffer, $1.25 \mu 1$ dNTPs, $0.5 \mu 1$ RNasin, $0.75 \mu 1$ M-MLV-RTase and nuclease-free water. RT-qPCR were performed on a BioRad Real-Time PCR platform using the following components: $10 \mu \mathrm{1} 2 \mathrm{X}$ SYBR Premix Ex-Taq, $0.8 \mu 1$ primers, $5 \mu \mathrm{l}$ cDNA and $4.2 \mu \mathrm{l} \mathrm{ddH}_{2} \mathrm{O}$. The primers used were as follows: Forward, 5'-ACCTGGGGAACAAAGGCT AC-3' and reverse, 5'-TAGGACCGAGAGGCAAAGAC-3' for ZFP91-P; forward, 5'-ATTCCACTTTGCGTTCAAGG-3' and reverse, 5'-CTTCAGAGAGAGGAAAGCCGA-3' for vimentin; forward, 5'-AGCTACTGCCTCCGGTCTTC-3' and reverse; 5'-GTGGTCAACAGCCAGCTCA-3' for $\beta$-catenin; and forward, 5'-GTGGACATCCGCAAAGAC-3' and reverse, 5'-AAAGGGTGTAACGCAACTA-3' for $\beta$-actin. The reaction conditions were an initial denaturation step at $95^{\circ} \mathrm{C}$ for $5 \mathrm{sec}$ and 40 cycles of denaturation at $95^{\circ} \mathrm{C}$ for $5 \mathrm{sec}$ followed by elongation at $60^{\circ} \mathrm{C}$ for $20 \mathrm{sec}$. Absorbance values obtained at the end of every elongation step were used to analyze fluorescence. Experiments were repeated at least three times and the comparative quantification cycle $\left(2^{-\Delta \Delta C q}\right)$ method (21) was used to analyze the relative mRNA expression levels of ZFP91-P.

MTT assay. Infected BXPC-3-H (3,000 cells/well) were reseeded in 96-well plates 4 days after lentivirus infection. The number of viable cells was measured at daily intervals (days 1, 2, 3, 4, and 5). At each time point, $10 \mu \mathrm{l}$ of $5 \mathrm{mg} / \mathrm{ml}$ MTT was added to the cells. After incubation for $4 \mathrm{~h}, 150 \mu \mathrm{l}$ acidic isopropanol (5\% isopropanol, $10 \% \mathrm{SDS}$ and $0.01 \mathrm{~mol} / \mathrm{l}$ $\mathrm{HCl})$ was added to dissolve the formazan crystals. The absorbance of each well was recorded at a wavelength of $595 \mathrm{~nm}$ using microplate reader.

Scratch migration assay. The scratch assay is a convenient and inexpensive method to analyze cell migration in vitro (22). Infected BXPC-3-H cells (1.0x104 cells/well) were seeded on 96-well plate at $37^{\circ} \mathrm{C}$ in a humidified incubator containing $5 \% \mathrm{CO} 2$ and, when cells were $>90 \%$ confluent, a scratch was made using the tip of $10 \mu \mathrm{l}$ sterile pipette. The initial scratch area and the degree of healing after scratching was observed under a binocular light microscope (Olympus $\mathrm{CH}-2$, Olympus Corp., Tokyo, Japan)after 12 and $48 \mathrm{~h}$.

Transwell migration assay. A Transwell ${ }^{\circledR}$ chamber (Corning, Inc, New York, NY, USA) was used to determine the migration ability of BXPC-3-H cells infected with target and control shRNA over $96 \mathrm{~h}$. shZFP91-P and shCon cells $\left(3.0 \times 10^{4}\right.$ cells/well) were seeded into the upper chamber in $100 \mu \mathrm{l}$ medium containing $0.1 \%$ FBS. Subsequently, $1 \mathrm{ml}$ medium, containing 10\% FBS as a chemoattractant, was added to the lower chamber. Then the migration system was placed in an incubator for $6 \mathrm{~h}$ at $37^{\circ} \mathrm{C}$ in $5 \% \mathrm{CO}_{2}$. Finally, the migrated cells were fixed with $4 \%$ paraformaldehyde and stained with crystal violet $(0.5 \%)$. Cell numbers were counted under a binocular light microscope in five random fields (magnification, x100) per filter and detected by the spectrometric absorbance at $570 \mathrm{~nm}$.

Western analysis. BXPC-3-H cells were collected $72 \mathrm{~h}$ post-infection with recombinant lentiviruses, washed in ice-cold phosphate-buffered saline and lysed in 2X SDS sample buffer. Total protein $(30 \mu \mathrm{g})$ was separated by electrophoresis using 10\% SDS-PAGE at $80 \mathrm{~V}$ for $30 \mathrm{~min}$ followed by $150 \mathrm{~V}$ for $1 \mathrm{~h}$. Subsequently, the separated protein samples were transferred onto a PVDF transmembrane (Millipore, Bedford, MA, USA) under $300 \mathrm{~mA}$ and blocked in $5 \%$ non-fat milk with Tris-buffered saline and $0.05 \%$ Tween 20 (Sigma, St. Louis, MO, USA) for $2 \mathrm{~h}$ at room temperature. The membrane was then incubated with following primary antibodies: Rabbit anti-vimentin (1:1,000 dilution; cat no. \#5741; 
A

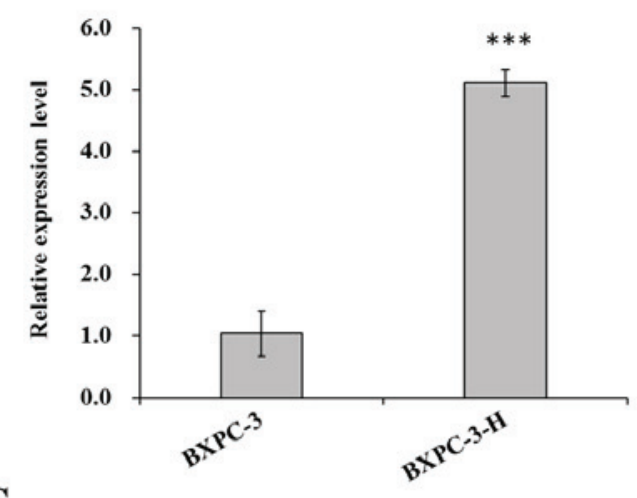

C

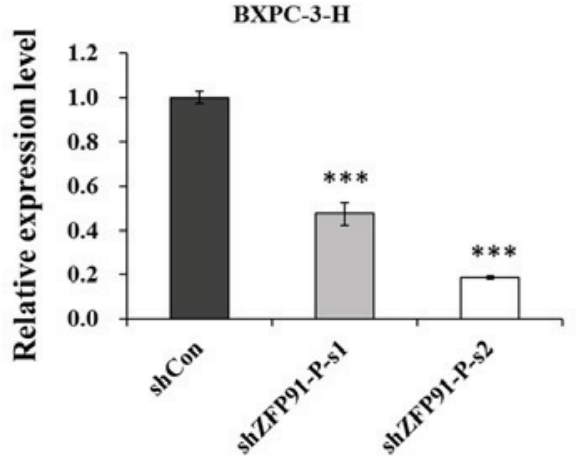

B

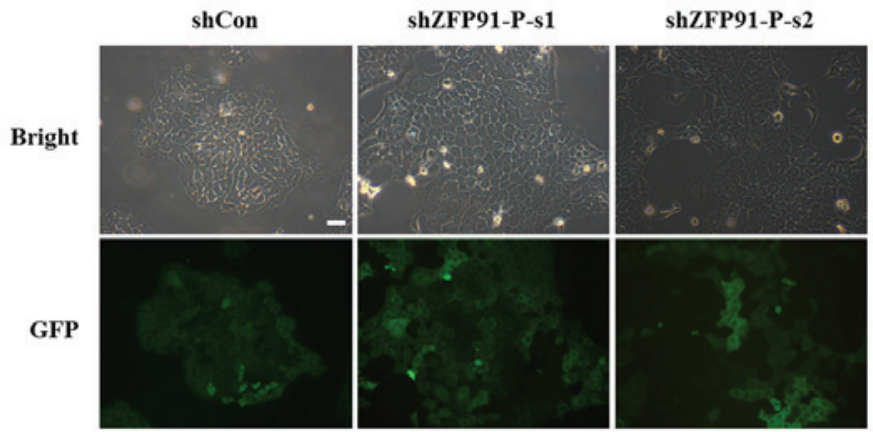

D

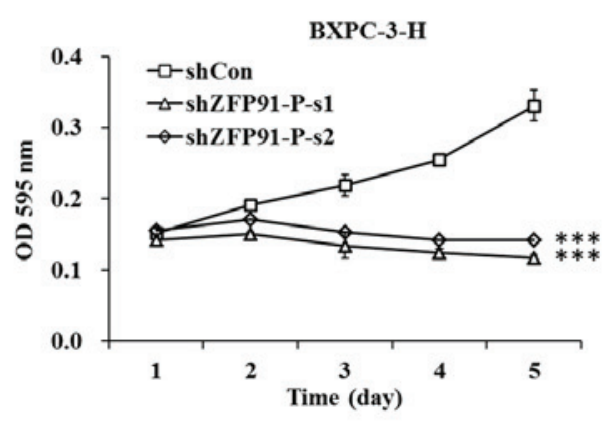

Figure 1. Expression of ZFP91-P in pancreatic cancer cells. (A) mRNA expression levels of ZFP91-P in BXPC-3 and BXPC-3-H cells were determined by reverse transcription-quantitative polymerase chain reaction (RT-qPCR). $\beta$-actin was used as an internal control gene. ${ }^{* * *} \mathrm{P}<0.001$ vs. $\mathrm{BXPC}-3$. (B) Fluorescence photomicrographs of BXPC-3-H cells infected by lentivirus. Multiplicity of infection, 20. Magnification, x100. Scale bar, $10 \mu$ m. (C) RT-qPCR analysis of ZFP91-P mRNA expression in BXPC-3-H cells with three treatments. $\beta$-actin was used as an internal control gene. ${ }^{* * *} \mathrm{P}<0.001 \mathrm{vs}$. shCon. (D) BXPC-3-H cell proliferation after ZFP91-P silencing, as determined by an MTT assay. Cells with three treatments including shCon, shZFP91-P-s1, and shZFP91-P-s2 groups shCon, BXPC-3-H cells infected with control shRNA; shZFP91-P-s1, BXPC-3-H cells infected with ZFP91-P shRNA 1; shZFP91-P-s2, BXPC-3-H cells infected with ZFP91-P shRNA2. ${ }^{* * *} \mathrm{P}<0.001$ vs. shCon. Data are presented as mean \pm standard error of the mean. sh, shRNA; Con, control; ZFP91-P, zinc finger protein 91 pseudogene; GFP, green fluorescent protein; OD, optical density.

Cell Signaling Technology, Danvers, MA, USA); rabbit anti- $\beta$-catenin (1:1,000 dilution; cat no. \#8480; Cell Signaling Technology) and rabbit anti-GAPDH (1:500,000 dilution; cat no. 10494-1-AP; Proteintech Group, Inc., Chicago, IL, USA) at $4{ }^{\circ} \mathrm{C}$ overnight, followed by incubation with horseradish peroxidase-conjugated secondary antibodies (1:5,000; cat no. SC-2054; Santa Cruz Biotechnology, Inc., Santa Cruz, CA, USA) for $2 \mathrm{~h}$ at room temperature. An ECL kit (Amersham; GE Healthcare Life Sciences) was used to visualize the blot.

Statistical analysis. All statistical data are expressed as mean \pm standard error of three independent experiments. Student's t-test was used to compare differences between groups. $\mathrm{P}<0.05$ was considered to indicate a statistically significant. Comparisons were carried out by Student's t-test and one-way ANOVA analysis using SPSS 22.0 statistical software (SPSS, Inc., Chicago, IL, USA).

\section{Results}

Expression of ZFP91-P is downregulated by Lv-shZFP91-P in pancreatic cancer cells. The expression of ZFP91-P was determined in two pancreatic cancer cell lines, BXPC-3 and BXPC-3-H. As shown in Fig. 1A, ZFP91-P mRNA levels were significantly upregulated in BXPC-3-H cells compared with in BXPC-3 cells $(\mathrm{P}<0.001)$. Thus, only BXPC-3-H cells were used in subsequent experiments. To clarify the biological function of ZFP91-P in pancreatic cancer cells, a lentivirus-mediated RNAi system was used to suppress ZFP91-P expression in BXPC-3-H cells. Fluorescence microscopy showed that $>80 \%$ of cells were GFP-positive in the Lv-shCon and Lv-shZFP91-P groups 3 days after lentivirus infection (Fig. 1B). Subsequently, RT-qPCR was used to analyze the knockdown efficiency of ZFP91-P. As shown in Fig. 1C, expression of ZFP91 was significantly inhibited by $52.5 \%$ in the Lv-shZFP91-P-s1 group and $81.4 \%$ in the Lv-shZFP91-P-s2 group compared with the Lv-shCon group, respectively $(\mathrm{P}=0.0006 ; \mathrm{P}=0.0002)$.

Knockdown of ZFP91-P significantly inhibits BXPC-3-H cell proliferation. To evaluate the effect of shZFP91-P on pancreatic cancer cell proliferation, an MTT assay was conducted in BXPC-3-H cells. The results showed that the growth curves of the LV-shZFP91-P group were significantly lower than those of the Lv-shCon group ( $\mathrm{P}<0.001$; Fig. 1D), suggesting that knockdown of ZFP91-P could significantly inhibit pancreatic cancer cell proliferation.

Depletion of ZFP91-P significantly inhibits the migratory ability of metastatic pancreatic cancer cells. A scratch migration assay was performed to evaluate the migration ability of 
A
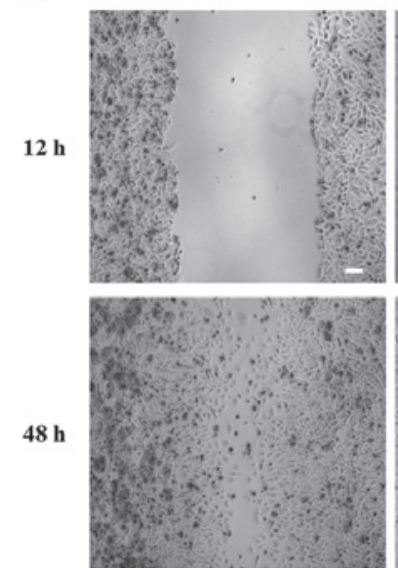

shZFP91-P

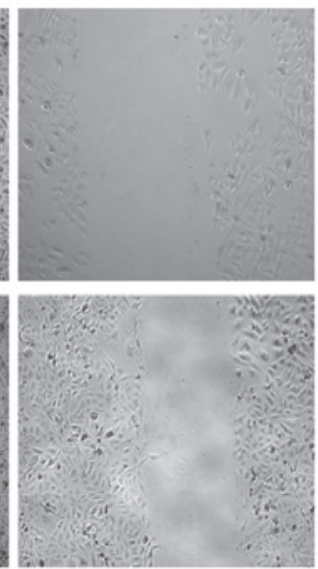

B

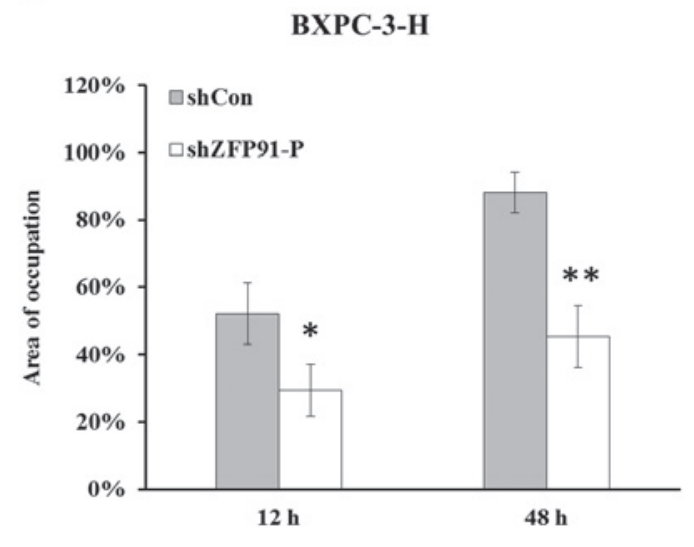

Figure 2. Scratch migration assay of the migratory ability of BXPC-3-H cells. (A) Images of the migration of BXPC-3-H cells when treated with shCon and shZFP91-P. Scale bar, $10 \mu \mathrm{m}$. Magnification, x40. (B) Percentage of wound confluence when pancreatic cancer cells were treated with different types of lentivirus. Data are presented as mean \pm standard error of the mean. ${ }^{*} \mathrm{P}<0.05,{ }^{* *} \mathrm{P}<0.01$ vs. shCon. sh, shRNA; Con, control; ZFP91-P, zinc finger protein 91 pseudogene.

A

shCon
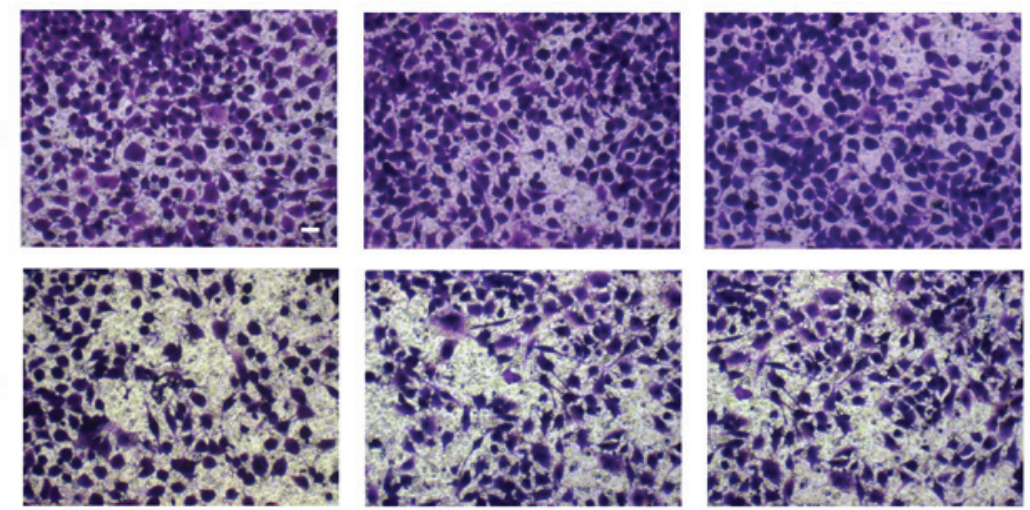

B

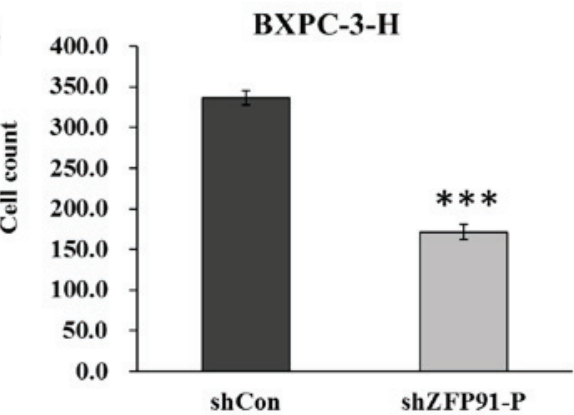

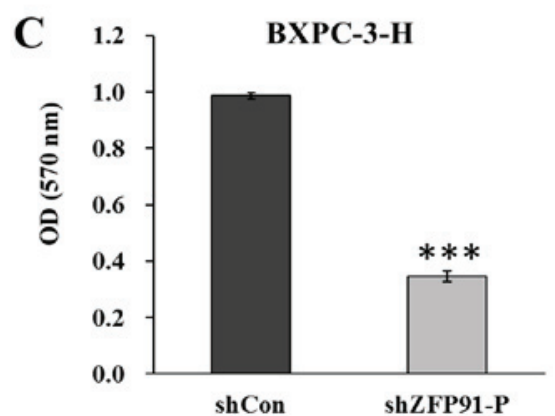

Figure 3. Transwell migration assay revealed that depletion of ZFP91-P resulted in decreased cell migration potential. (A) Microscopic images of migrated BXPC-3-H cells at $6 \mathrm{~h}$ (3 replicates). Crystal violet staining. Scale bar, $10 \mu \mathrm{m}$. Magnification, $\mathrm{x} 40$. (B) Number of migrated cells. (C) Quantitative analysis of stained migrated cancer cells using a microplate reading at an $\mathrm{OD}$ at $570 \mathrm{~nm}$. Data are presented as mean \pm standard error of the mean. ${ }^{* * *} \mathrm{P}<0.001 \mathrm{vs}$. shCon. sh, shRNA; Con, control; ZFP91-P, zinc finger protein 91 pseudogene; OD, optical density.

BXPC-3-H cells in vitro. At the selected time points of 12 and $48 \mathrm{~h}$, cell migration distance was observed under a microscope (magnification, x100) (Fig. 2A). Cell migration ability was significantly inhibited in the Lv-shZFP91-P group compared with the Lv-shCon group, with control BXPC-3-H cells showing a better ability to heal the scratch $(\mathrm{P}<0.05)$ (Fig. 2B). Subsequently, a Transwell assay was used to determine the effect of ZFP91-P knockdown in regulating pancreatic cancer cell migration (Fig. 3A). As indicated in Fig. 3B, significantly fewer cells in the Lv-shZFP91-P group migrated to the lower surface of the membrane, compared with cells in the Lv-shCon group $(\mathrm{P}<0.001)$. In addition, the crystal violet staining intensity was significantly lower in the Lv-shZFP91-P group than in the Lv-shCon group $(\mathrm{P}<0.001)$ (Fig. $3 \mathrm{C})$. These results suggest that ZFP91-P may have a key role in pancreatic cancer metastasis.

Downregulation of ZFP91-P elevates the expression of $\beta$-catenin and inhibits the expression of vimentin via epithelial-mesenchymal transition (EMT) signaling. To 
A

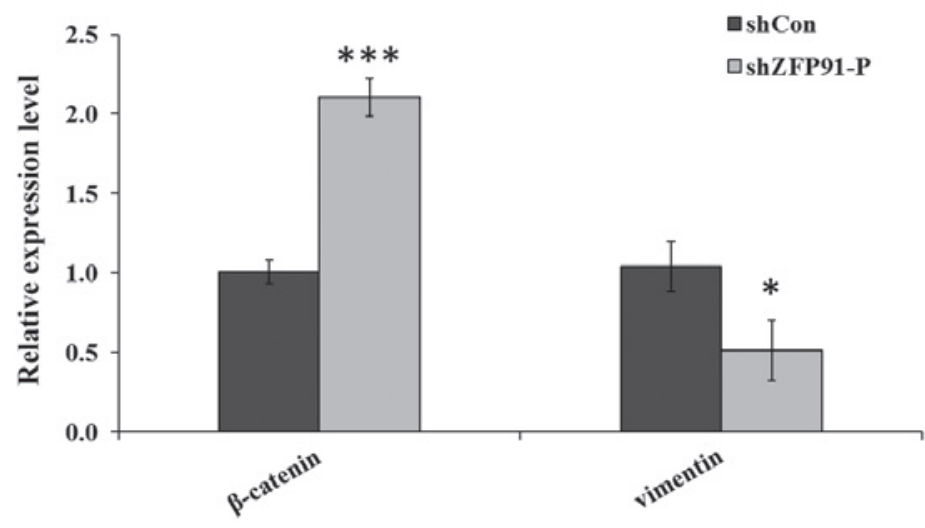

B

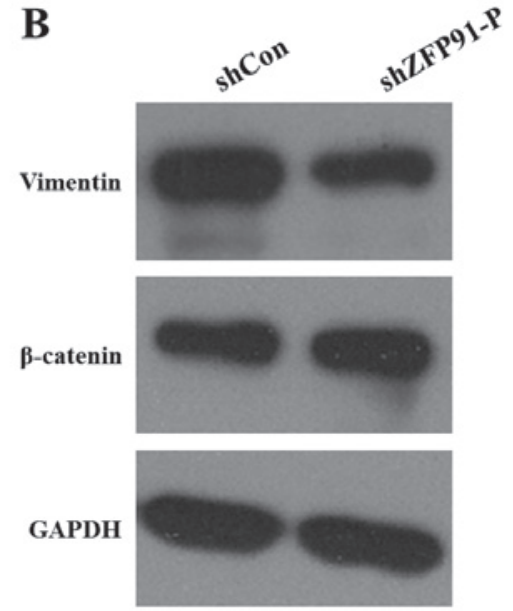

Figure 4. Relative mRNA and protein expression level of $\beta$-catenin and vimentin. (A) mRNA expression levels of $\beta$-catenin and vimentin in BXPC-3-H cells were determined by reverse transcription-quantitative polymerase chain reaction. Data are presented as mean \pm standard error of the mean. ${ }^{*} \mathrm{P}<0.05,{ }^{* * *} \mathrm{P}<0.001$ vs. shCon. (B) Protein expression levels of $\beta$-catenin and vimentin in BXPC-3-H cells were determined by western blotting. sh, shRNA; Con, control; ZFP91-P, zinc finger protein 91 pseudogene.

evaluate the regulatory role of ZFP91-P in EMT signaling in human pancreatic cancer, the effect of ZFP91-P silencing on the expression of $\beta$-catenin and vimentin, which are critical for tumor invasion and metastasis, was examined. RT-qPCR results showed that the mRNA expression levels of $\beta$-catenin were significantly elevated $(\mathrm{P}<0.001)$ while vimentin was significantly reduced $(\mathrm{P}<0.05)$ in the Lv-shZFP91-P group compared with the Lv-shCon group in BXPC-3-H cells (Fig. 4A). Analysis of protein expression levels revealed the same pattern (Fig. 4B).

\section{Discussion}

Pancreatic cancer is one of the most fatal neoplastic diseases, as it is typically diagnosed at an advanced stage. The majority of cases of pancreatic cancer are inoperable and metastasized, therefore, it is difficult to overcome this health problem. Thus far, numerous gene abnormalities have been found to be involved in pancreatic cancer (23-25). Gene therapy is designed to deliver a therapeutic gene into a target site to regulate expression of the specific gene (26-28).

It has been reported that ZFP91 may have an important role in cell proliferation (17) and may be involved in prostate cancer (29). Several transcribed pseudogenes, such as PTENP1, KRASP1 and OCT4-pg4, have been known to promote tumor progression $(13,14)$. These findings prompted the present study to investigate the ZFP91-P gene as a target site in pancreatic cancer therapy.

In the present study, the association between ZFP91-P and characteristics of pancreatic cancer were initially examined. ZFP91-P showed high expression in BXPC-3-H and BXPC-3 pancreatic cancer cells. Subsequently, the expression of ZFP91-P was knocked down in BXPC-3-H cells using a lentivirus-based shRNA system. The cell proliferation and migration ability were impaired in the absence of ZFP91-P, suggesting that ZFP91-P may promote the metastatic and motility properties of pancreatic cancer cells.

EMT is a biological process that can enhance cell migratory capacity and invasiveness. It is clear that EMT occurs in three distinct biological settings, including organ development (30), tumor growth and cancer progression (31). Furthermore, it has been reported that EMT acts as a major driver of tumor invasion (32), with a crucial role in the aggressiveness and invasion of pancreatic cancer (33). The expression of mesenchymal makers, such as ZEB1, vimentin, Slug and Snail, are positive for majority of cases of pancreatic cancer (34). To further clarify these results, the present study identified the signaling elements targeted by ZFP91-P for the promotion of EMT in pancreatic cancer. ZFP91-P silencing appeared to reverse EMT, as shown by increased expression of $\beta$-catenin and decreased expression of vimentin. Vimentin, a major component of the intermediate filament family, has been demonstrated to markedly reverse EMT in pancreatic cancer cells, acting as a mesenchymal marker (35). In addition, its overexpression may result in accelerated cell growth, invasion and poor prognosis in numerous types of cancer (36). The current results indicate that ZFP91-P silencing can inhibit cell proliferation and migration by reversing the EMT pathway. $\beta$-catenin not only has a critical role in cell-cell adhesion by interacting with cadherins at the plasma membrane, but is also involved in a signaling cascade at the center of the Wnt signaling pathway. Several previous studies have demonstrated that $\beta$-catenin is essential for normal pancreatic organogenesis $(37,38)$. However, stable expression of $\beta$-catenin within the pancreatic epithelium can give rise to the formation of tumors $(39,40)$. By contrast, concurrent activation of $\beta$-catenin and Kras prevents the formation of pancreatic intraepithelial neoplasias (40). In addition, increased $\beta$-catenin activity by overexpression of OCT-4 can inhibit cell differentiation (40). Based on these points, we propose that $\beta$-catenin may be concurrently activated with certain genes during ZFP91-P silencing in pancreatic cancer cells. However, further studies are required to investigated the molecular mechanism of $\beta$-catenin in pancreatic cancer.

In conclusion, the current study indicates that ZFP91-P is an important regulator of pancreatic cancer cell migration and proliferation, and ZFP91-P silencing may suppress the migration of pancreatic cancer cells by reversing EMT. Therefore, 
ZFP91-P may be a useful target for gene therapy in pancreatic cancer. However, the biological behavior of ZFP91-P in pancreatic cancer needs to be thoroughly explored in future studies.

\section{Acknowledgements}

The present study was funded by the Shanghai Municipal Public Health Bureau (Shanghai, China), grant no. 20114178.

\section{References}

1. Siegel R, Ward E, Brawley O and Jemal A: Cancer statistics, 2011: The impact of eliminating socioeconomic and racial disparities on premature cancer deaths. CA Cancer J Clin 61: 212-236, 2011.

2. Ma J, Siegel R and Jemal A: Pancreatic cancer death rates by race among US men and women, 1970-2009. J Natl Cancer Inst 105 $1694-1700,2013$

3. Li D, Xie K, Wolff R and Abbruzzese JL: Pancreatic cancer. Lancet 363: 1049-1057, 2004.

4. Jemal A, Siegel R, Ward E, Hao Y, Xu J, Murray T and Thun MJ: Cancer statistics, 2008. CA Cancer J Clin 58: 71-96, 2008.

5. Rozenblum E, Schutte M, Goggins M, et al: Tumor-suppressive pathways in pancreatic carcinoma. Cancer Res 57: 1731-1734, 1997

6. Luo J, Guo P, Matsuda K, et al: Pancreatic cancer cell-derived vascular endothelial growth factor is biologically active in vitro and enhances tumorigenicity in vivo. Int J Cancer 92: 361-369, 2001.

7. Xie K: Interleukin- 8 and human cancer biology. Cytokine Growth Factor Rev 12: 375-391, 2001.

8. Yamanaka Y, Friess H, Buchler M, et al: Overexpression of acidic and basic fibroblast growth factors in human pancreatic cancer correlates with advanced tumor stage. Cancer Res 53: 5289-5296, 1993.

9. Ponting CP, Oliver PL and Reik W: Evolution and functions of long noncoding RNAs. Cell 136: 629-641, 2009.

10. Wilusz JE, Sunwoo H and Spector DL: Long noncoding RNAs: Functional surprises from the RNA world. Genes Dev 23 1494-1504, 2009.

11. Grandér D and Johnsson P: Pseudogene-expressed RNAs: Emerging roles in gene regulation and disease. Curr Top Microbiol Immunol, 2015.

12. Welch JD, Baran-Gale J, Perou CM, Sethupathy P and Prins JF Pseudogenes transcribed in breast invasive carcinoma show subtype-specific expression and ceRNA potential. BMC Genomics 16: 1-16, 2015.

13. Poliseno L, Salmena L, Zhang J, Carver B, Haveman WJ and Pandolfi PP: A coding-independent function of gene and pseudogene mRNAs regulates tumour biology. Nature 465 1033-1038, 2010.

14. Hayashi H, Arao T, Togashi Y, Kato H, Fujita Y, De Velasco MA, Kimura H, Matsumoto K, Tanaka K, Okamoto I, et al: The OCT4 pseudogene POU5F1B is amplified and promotes an aggressive phenotype in gastric cancer. Oncogene 34: 199-208, 2015.

15. Vanaja DK, Cheville JC, Iturria SJ and Young CY: Transcriptional silencing of zinc finger protein 185 identified by expression profiling is associated with prostate cancer progression. Cancer Res 63: 3877-3882, 2003.

16. Kiem HP, Ironside C, Beard BC and Trobridge GD: A retroviral vector common integration site between leupaxin and zinc finger protein 91 (ZFP91) observed in baboon hematopoietic repopulating cells. Exp Hematol 38: 819-822, 2010.

17. Unoki M, Okutsu J and Nakamura Y: Identification of a novel human gene, ZFP91, involved in acute myelogenous leukemia. Int J Oncol 22: 1217-1223, 2003.

18. Al Dulaimi D: Recent advances in gastric cancer. Gastroenterol Hepatol Bed Bench 7: 238-240, 2014.

19. Kim DH, Behlke MA, Rose SD, Chang MS, Choi S and Rossi JJ: Synthetic dsRNA Dicer substrates enhance RNAi potency and efficacy. Nat Biotechnol 23: 222-226, 2005.
20. Guo W, Zhang Y, Chen T, Wang Y, Xue J, Zhang Y, Xiao W, Mo X and Lu Y: Efficacy of RNAi targeting of pyruvate kinase M2 combined with cisplatin in a lung cancer model. J Cancer Res Clin Oncol 137: 65-72, 2011.

21. Livak KJ and Schmittgen TD: Analysis of relative gene expression data using real-time quantitative PCR and the 2 (-Delta Delta C (T)) method. Methods 25: 402-408, 2001.

22. Liang CC, Park AY and Guan JL: In vitro scratch assay: A convenient and inexpensive method for analysis of cell migration in vitro. Nat Protoc 2: 329-333, 2007.

23. Shain AH, Giacomini CP, Matsukuma K, Karikari CA, Bashyam MD, Hidalgo M, Maitra A and Pollack JR: Convergent structural alterations define SWItch/Sucrose NonFermentable (SWI/SNF) chromatin remodeler as a central tumor suppressive complex in pancreatic cancer. Proc Natl Acad Sci USA 109: E252-E259, 2012.

24. Jones S, Zhang X, Parsons DW, Lin JC, Leary RJ, Angenendt P, Mankoo P, Carter H, Kamiyama H, Jimeno A, et al: Core signaling pathways in human pancreatic cancers revealed by global genomic analyses. Science 321: 1801-1806, 2008

25. Efthimiou E, Crnogorac-Jurcevic T, Lemoine NR and Brentnall TA Inherited predisposition to pancreatic cancer. Gut 48: 143-147, 2001.

26. Chang H: RNAi-mediated knockdown of target genes: A promising strategy for pancreatic cancer research. Cancer Gene Ther 14: 677-685, 2007.

27. Gartel AL and Kandel ES: RNA interference in cancer. Biomol Eng 23: 17-34, 2006.

28. Gleich LL: Gene therapy for head and neck cancer. Laryngoscope 110: 708-726, 2000.

29. Paschke L, Rucinski M, Ziolkowska A, Zemleduch T, Malendowicz W, Kwias Z and Malendowicz LK: ZFP91-a newly described gene potentially involved in prostate pathology. Pathol Oncol Res 20: 453-459, 2014.

30. Zeisberg M, Hanai J, Sugimoto H, Mammoto T, Charytan D, Strutz F and Kalluri R: BMP-7 counteracts TGF-beta1-induced epithelial-to-mesenchymal transition and reverses chronic renal injury. Nat Med 9: 964-968, 2003.

31. Thiery JP: Epithelial-mesenchymal transitions in tumour progression. Nat Rev Cancer 2: 442-454, 2002.

32. Thiery JP, Acloque H, Huang RY and Nieto MA: Epithelial-mesenchymal transitions in development and disease. Cell 139: 871-890, 2009.

33. Kurahara H, Takao S, Maemura K, Mataki Y, Kuwahata T, Maeda K, Ding Q, Sakoda M, Iino S, Ishigami S, et al: Epithelial-mesenchymal transition and mesenchymal-epithelial transition via regulation of ZEB-1 and ZEB-2 expression in pancreatic cancer. J Surg Oncol 105: 655-661, 2012.

34. Hotz B, Arndt M, Dullat S, Bhargava S, Buhr HJ and Hotz HG: Epithelial to mesenchymal transition: Expression of the regulators snail, slug, and twist in pancreatic cancer. Clin Cancer Res 13: 4769-4776, 2007.

35. Wang YL, Dong FL, Yang J, Li Z, Zhi QM, Zhao X, Yang Y, Li DC, Shen XC and Zhou J: Suppression of the epidermal growth factor-like domain 7 and inhibition of migration and epithelial-mesenchymal transition in human pancreatic cancer PANC-1 cells. Asian Pac J Cancer Prev 16: 4065-4069, 2015

36. Satelli A and Li S: Vimentin in cancer and its potential as a molecular target for cancer therapy. Cell Mol Life Sci 68: 3033-3046, 2011.

37. Murtaugh LC, Law AC, Dor Y and Melton DA: Beta-catenin is essential for pancreatic acinar but not islet development. Development 132: 4663-4674, 2005.

38. Wells JM, Esni F, Boivin GP, Aronow BJ, Stuart W, Combs C, Sklenka A, Leach SD and Lowy AM: Wnt/beta-catenin signaling is required for development of the exocrine pancreas. BMC Dev Biol 7: 4, 2007

39. Hochedlinger K, Yamada Y, Beard C and Jaenisch R: Ectopic expression of Oct-4 blocks progenitor-cell differentiation and causes dysplasia in epithelial tissues. Cell 121: 465-477, 2005.

40. Heiser PW, Cano DA, Landsman L, Kim GE, Kench JG, Klimstra DS, Taketo MM, Biankin AV and Hebrok M: Stabilization of beta-catenin induces pancreas tumor formation. Gastroenterology 135: 1288-1300, 2008. 